\title{
Relationship between validity of center of gravity transferring velocities measured by rotary encoder and three-dimensional motion analysis during sit-to-stand movement in the elderly
}

\author{
Takayoshi Yamada ${ }^{*}$, Shinichi Demura \\ Faculty of Education and Regional Studies, University of Fukui, Fukui, Japan \\ Graduate School of Natural Science \& Technology, Kanazawa University, Ishikawa, Japan \\ *Corresponding author E-mail: yamadat@u-fukui.ac.jp
}

\begin{abstract}
This study examined the validity of center of gravity transferring velocity (VCG) measured by a rotary encoder (RE) during sit-to-stand (STS) movement with that measured by three-dimensional motion analysis (3DMA). Fifteen healthy elderly females (age, 78.7 \pm 4 .6 years) were asked to stand up as fast as possible without assistance from a chair adjusted for their knee height. VCG was measured simultaneously by 3DMA and RE. The peak and mean velocities from the start to movement completion were calculated by both methods. Cross, crosswise, and vertical directions were also calculated for the 3DMA. Significant relationships were found between peak and mean velocities measured by RE and the cross and vertical velocities measured by the 3DMA (peak velocity: cross, 0.60 , vertical, 0.97 ; mean velocity: cross, 0.77 , vertical, 0.75), but low in crosswise $(\mathrm{r}<0.47)$. RE is useful for evaluating VCG during STS movement and is highly correlated with the vertical velocity measured by 3DMA.
\end{abstract}

Keywords: center of gravity, practicability, sit-to-stand, 3-dimentional motion analysis, validity

\section{Introduction}

Physical function in the elderly should be conveniently measured and evaluated using activities of daily living (ADL), and the results of these measurements should be presented to patients in an easy-to-understand form. Functional tests using ADL such as gait and stair-stepping have been proposed. Because sit-to-stand (STS) movement precedes ambulatory and other basic ADL [1], a stable and smooth STS movement is indispensable for maintaining an independent life [2]. Therefore, the importance of evaluating the achievement ability of the STS movement has been advocated until now, and also the test to evaluate it has been developed.

The STS movement is defined as an upward transfer of the center of gravity [3,4]. It is divided into two movement phases - the trunk flexion and the knee and hip joint extension. The former is the forward (horizontal) transfer of the center of gravity from movement initiation until lifting of the hips off the chair seat, and the latter is the phase between the hip lift and the standing posture with extension of the hips and both knee joints. The elderly require a certain level of leg strength and balance to perform the STS movement with stability, rapidity, and smoothness.

The STS movement has been used not only as a measure of decreased functionality with age but also as an index of improvement with training. Schot et al. [5] conducted fitness conditioning for the elderly based on a general strengthtraining design and reported that maximal forward-, upward-, and downward-transferring velocities of the center of gravity and the time required improved significantly (increased velocities, 16\%, 59\%, and 26\%, respectively; decreased time, $27 \%$ ). Stability of movement also improved with training.

The position and displacement of the center of gravity have generally been measured by three-dimensional (3D) motion analysis [5-13]. However, determining the center-of-gravity positions by this method is very difficult for clinical applications. Thus, the development of an alternative measurement method that can conveniently and adequately evaluate the center of gravity transferring velocity during the STS movement is required.

We previously proposed a convenient method for evaluating the center of gravity transferring velocity during the STS movement using a rotary encoder [14]. This method measures and evaluates the length of a pulled or returned cord while performing the STS movement with a belt placed at the crista iliaca height. Good reliability and relationship with 
leg strength were observed when this method was tested on young adults. However, its relationship with 3D motion analysis, as the gold standard for determining the center of gravity, has not been previously investigated and is required to clarify the accuracy of this simple method.

Thus, the present study aimed to examine the validity of the center of gravity transferring velocity during the STS movement measured by the rotary encoder compared with that measured by 3D motion analysis.

\section{Methods}

\subsection{Subjects}

Fifteen elderly females living independently (age, $78.7 \pm 4.6$ years; height, $147.7 \pm 6.7 \mathrm{~cm}$; body mass, $48.7 \pm 6.6 \mathrm{~kg}$ ) participated in this study. Written informed consent was obtained from all subjects after a full explanation of the experimental purpose and protocol. The study protocol was approved by the Ethics Committee on Human Experimentation of Faculty of Human Science, Kanazawa University (authorization number: 2012-04).

\subsection{Materials}

The center of gravity transferring velocities were measured simultaneously by 3D motion analysis and the rotary encoder. A Kinema Tracer (Kisseicomtec Co., Ltd.) was used for 3D motion analysis. Four video cameras were used as trace markers and were placed on the acromiale, upper end of greater trochanter, lateral supracondylar ridge, lateral malleolus, and fifth metatarsal bone, with reference to a previous study [15]. The center-of-gravity position was calculated by the recorded displacement of each marker, and cross, crosswise, and the vertical center of gravity transferring velocities were calculated. Data was recorded at $100 \mathrm{~Hz}$ on a personal computer.

The center of gravity transferring velocities during the STS movement were also measured by a FiTRO Dyne Premium (FiTRONiC s.r.o., Bratislava, Slovakia). This device measures the length of a pulled or returned cord from a bobbin with a built-in rotary encoder. A belt was placed at the crista iliaca height. The cord was fixed at the left crista iliaca position on the belt, and the length of the pulled or returned cord was measured over time as each subject performed the STS movement. The center-of-gravity position was located in the abdominal region in the sitting posture, transferred from the abdominal to the lumbar position during the movement, and stabilized at the lumber position [16]. The crista iliaca-transferring velocities measured by the change in the pulled or returned cord length over time is assumed to reflect the center of gravity transferring velocities during the STS movement. Data were recorded at $100 \mathrm{~Hz}$ on a personal computer.

\subsection{Procedures}

For measuring the center of gravity transferring velocities, the subjects sat on a chair that was adjusted to their knee height in the following posture: legs were placed shoulder-width apart with bare feet; the trunk was in a straight line; ankle angle was at $90^{\circ}$; and arms were folded across the front of the chest. The subjects were instructed to stand up from the sitting posture as fast as possible at the tester's signal. After adequate explanation and one practice trial, the center of gravity transferring velocities during the STS movement were measured by 3D motion analysis and the rotary encoder.

\subsection{Parameters}

Figures 1 and 2 show representative examples of the time-course changes in the center of gravity transferring velocities during the STS movement measured in this study. Two parameters - the peak and mean velocities from movement initiation to completion - were selected using the time-course data. In addition, the time-course data obtained from 3D motion analysis was divided into three components - the cross, crosswise, and vertical directions. These parameters were calculated for each component and used for analysis.

\subsection{Statistical analysis}

Cross-correlation coefficients were calculated using the time-course data of the center of gravity transferring velocities during the STS movement measured by 3D motion analysis and the rotary encoder, and lags were calculated for the highest correlations. Intraclass correlation coefficients were calculated to examine trial-to-trial reliability of the peak and mean center of gravity transferring velocities during the STS movement. Pearson's correlation coefficients were 
calculated to examine the relationships between the peak and mean velocities measured by 3D motion analysis and the rotary encoder. A p value of $<0.05$ was indicative of statistical significance.

\section{Results}

Figure 1 shows a representative example of the time-course changes in the cross-, crosswise-, and vertical-direction transferring distances and velocities during the STS movement measured by 3D motion analysis. Cross- and verticaldirection transferring distances of the center of gravity increased with the starting movement and reached the peak values at the completion of movement. Large changes were not found in the crosswise-direction transferring distance. Moreover, the center of gravity transferring velocities of the cross and vertical directions showed a sharp increase with the starting movement and decreased after reaching a peak value. However, a similar trend in the crosswise velocity was not found in addition to not achieving a large peak value.
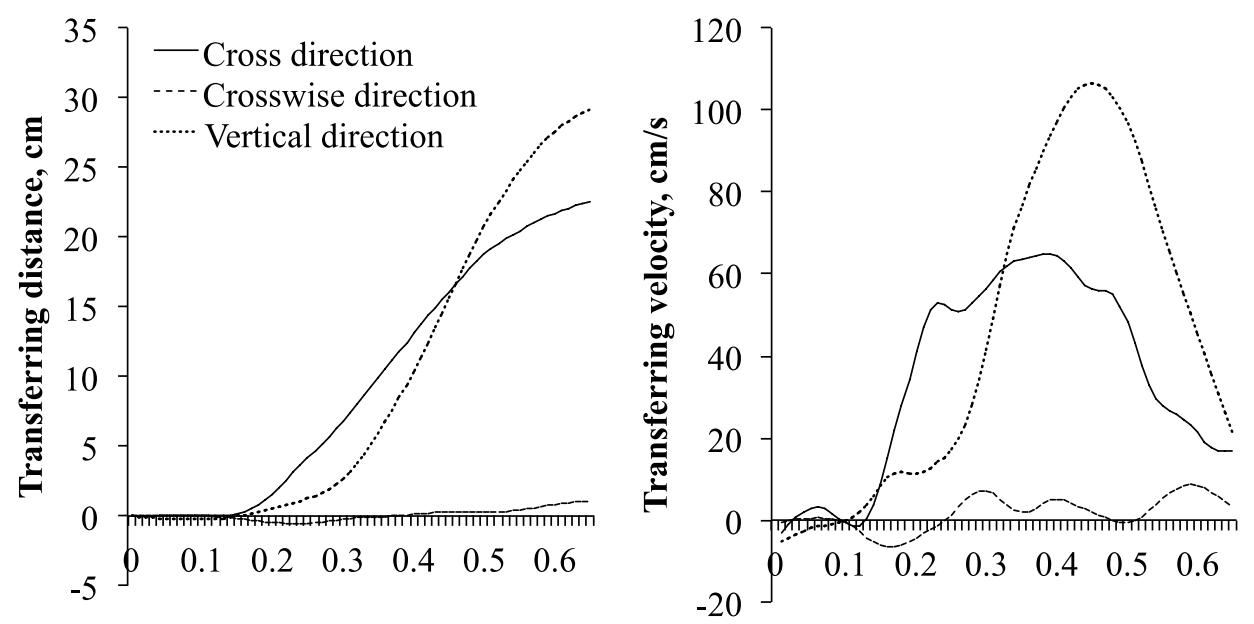

Fig. 1: Representative examples of the time-course change in the cross, crosswise, and vertical directions transferring distances (left) and velocities (right) during the STS movement measured by three-dimensional motion analysis

Figure 2 shows a representative example of the time-course changes in transferring distances and velocities during the STS movement measured by the rotary encoder. Transferring distance increased with the starting movement and reached a peak value at movement completion. The transferring velocity increased sharply with the starting movement and decreased after reaching a peak value. The transferring distances and velocities measured by the rotary encoder were very similar to the changes in the distance and velocity in the vertical direction measured by the $3 \mathrm{D}$ motion analysis.
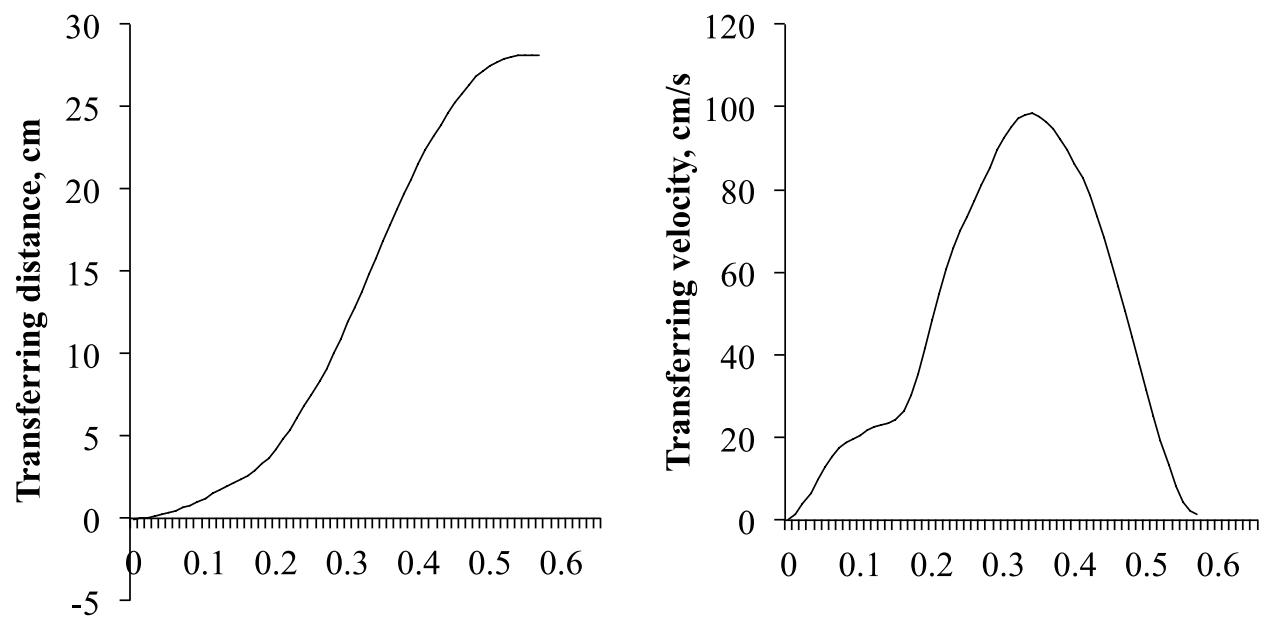

Fig. 2: Representative examples of the time-course change in transferring distances (left) and velocities (right) during the STS movement measured by the rotary encoder 
Table 1 shows cross correlations among the time-course data of the center of gravity transferring velocities during the STS movement measured by 3D motion analysis and the rotary encoder. Significant relationships were found between the center of gravity transferring velocities during the STS movement measured by the rotary encoder and all components measured by 3D motion analysis (cross direction, 0.58-0.99; crosswise direction, 0.66-0.97; vertical direction, 0.83-0.99).

Table 1: Cross correlations among the time-course data of the center-of-gravity transferring velocities during the STS movement measured by threedimensional motion analysis and the rotary encoder

\begin{tabular}{|c|c|c|c|c|c|c|c|c|c|}
\hline & & \multicolumn{4}{|c|}{$\mathrm{r}$} & \multicolumn{4}{|c|}{ lag } \\
\hline & & Max & & Min & & Mean & SD & Max & Min \\
\hline \multirow{3}{*}{$\begin{array}{l}\text { 3D motion } \\
\text { analysis }\end{array}$} & Cross direction & 0.99 & $*$ & 0.58 & $*$ & -27.87 & 13.28 & 6 & -37 \\
\hline & $\begin{array}{l}\text { Crosswise } \\
\text { direction }\end{array}$ & 0.97 & $*$ & 0.66 & $*$ & -15.53 & 20.14 & 28 & -35 \\
\hline & $\begin{array}{l}\text { Vertical } \\
\text { direction }\end{array}$ & 0.99 & * & 0.83 & * & 24.42 & 6.45 & 36 & 14 \\
\hline
\end{tabular}

Table 2 shows the trial-to-trial reliability coefficients in the peak and mean center of gravity velocities during the STS movement measured by 3D motion analysis and the rotary encoder. All reliability coefficients of the peak and mean center of gravity velocities measured by 3D motion analysis were good (ICC $=0.78-0.94)$, except for the peak velocity in the crosswise direction (ICC $=0.54$ ). Reliability coefficients of the peak and mean center of gravity transferring velocities measured by the rotary encoder were all good $(\mathrm{ICC}=0.77$ and 0.81$)$.

Table 2: Trial-to-trial reliability coefficients in the peak and mean velocities of the center of gravity during the STS movement as measured by threedimensional motion analysis and the rotary encoder

\begin{tabular}{|c|c|c|c|c|c|c|c|c|}
\hline & & & \multicolumn{3}{|c|}{ Peak velocity } & \multicolumn{3}{|c|}{ Mean velocity } \\
\hline & & & 1st trial & 2nd trial & ICC & 1st trial & 2nd trial & ICC \\
\hline \multirow{6}{*}{$\begin{array}{l}\text { 3D motion } \\
\text { analysis }\end{array}$} & \multirow{2}{*}{$\begin{array}{l}\text { Cross } \\
\text { direction }\end{array}$} & Mean & 49.04 & 52.58 & \multirow{2}{*}{0.78} & 24.55 & 25.33 & \multirow{2}{*}{0.89} \\
\hline & & SD & 11.56 & 8.45 & & 3.98 & 3.97 & \\
\hline & \multirow{2}{*}{$\begin{array}{l}\text { Crosswise } \\
\text { direction }\end{array}$} & Mean & 5.85 & 5.56 & \multirow{2}{*}{0.54} & 0.39 & 0.45 & \multirow{2}{*}{0.79} \\
\hline & & SD & 1.92 & 2.57 & & 1.26 & 1.87 & \\
\hline & \multirow{2}{*}{$\begin{array}{l}\text { Vertical } \\
\text { direction }\end{array}$} & Mean & 84.33 & 88.32 & \multirow{2}{*}{0.94} & 23.75 & 22.88 & \multirow{2}{*}{0.91} \\
\hline & & SD & 24.30 & 23.05 & & 7.43 & 5.85 & \\
\hline \multirow{2}{*}{$\begin{array}{l}\text { Rotary } \\
\text { encoder }\end{array}$} & & Mean & 69.65 & 74.44 & \multirow[b]{2}{*}{0.77} & 37.79 & 34.72 & \multirow[b]{2}{*}{0.81} \\
\hline & & SD & 18.11 & 19.82 & & 12.23 & 9.63 & \\
\hline
\end{tabular}

Figure 3 shows relationships between the peak and mean velocities in cross, crosswise, and vertical directions measured by $3 \mathrm{D}$ motion analysis and the rotary encoder. The peak and mean velocities in the crosswise direction during the STS movement measured by 3D motion analysis showed a low correlation with that measured by the rotary encoder $(\mathrm{r}=$ 0.04 and -0.47$)$. However, all other correlations were moderate or above $(r=0.60-0.97)$. In particular, a very high correlation was found between the peak velocities in the vertical direction $(r=0.97)$. 

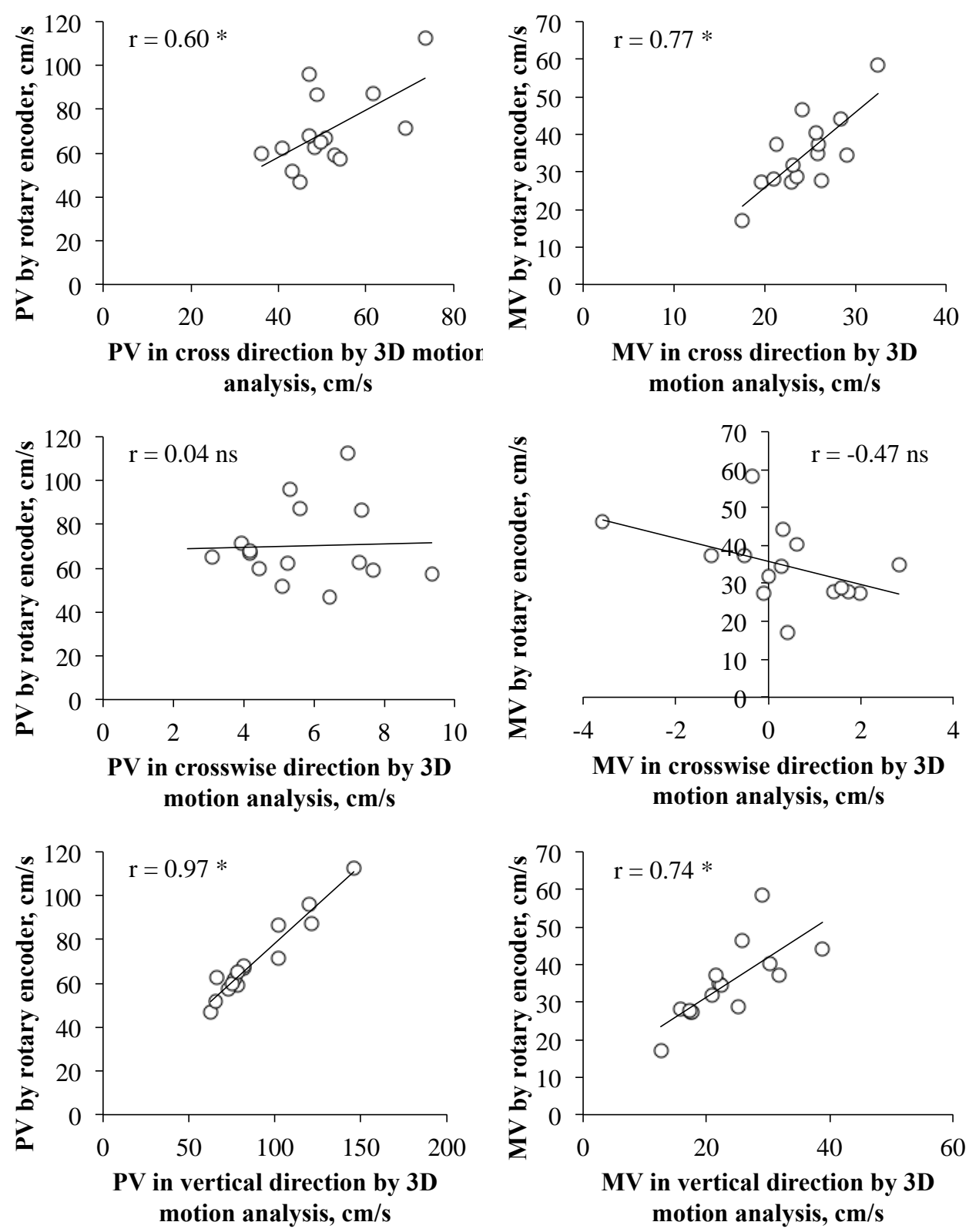

Fig. 3: Relationships between the peak and mean velocities in the cross, crosswise, and vertical directions measured by three-dimensional motion analysis and those measured by the rotary encoder: PV =

\section{Discussion}

The characteristics of the center of gravity transfer during the STS movement are largely divided into cross and vertical directions. The cross direction is the phase in which the hips lift off the chair seat after trunk flexion with the starting movement, and the vertical direction is that in which the knee and hip joints extend and the standing posture is then reached. This simple method using the rotary encoder proposed in this study reflects the synthesis of the rates of the cross and vertical components of the transferring velocity in the crista iliaca during the STS movement from sitting to standing posture. Values measured by 3D motion analysis can adequately evaluate the phased processes of the STS movement.

The center of gravity transferring velocities during the STS movement measured by both 3D motion analysis and the rotary encoder increased with the starting movement and decreased sharply after reaching a peak value. In particular, these trends were marked for velocities in the cross and vertical directions measured by 3D motion analysis, and the pattern of change in the vertical velocity measured by both methods were very similar. In addition, the center of gravity 
transferring velocities during the STS movement measured by both methods showed similar patterns to the cross and vertical directions of the center of gravity during the STS movement reported by Hirschfeld et al. [6] Although all relationships between each component velocity measured by 3D motion analysis and velocity measured by the rotary encoder were relatively high, lags were observed. These lags are considered to reflect differences in the abovemeasured values.

All reliability coefficients of the peak and mean velocities during the STS movement with both measurement methods were relatively high ( $3 \mathrm{D}$ motion analysis, ICC $=0.79-0.94$; rotary encoder, ICC $=0.77$ and 0.81 ). We previously examined the trial-to-trial reliability of the peak and mean center of gravity transferring velocities during the STS movement in young adults using the same simple method as that used in this study and reported very high reliability coefficients ( $\mathrm{ICC}=0.92$ and 0.85 , respectively [14]). The elderly require leg strength and balance above a certain level to achieve the STS movement with stability, rapidity, and smoothness because STS requires moving in different directions in the two half-phases. Furthermore, the STS movement becomes slower and less stable in old age because of the decrease in physical functioning. Therefore, trial-to-trial reliability of the peak and mean center of gravity transferring velocities during the STS movement are inferred to be lower in the elderly than in youth. However, the high reliability in the present study is similar to that previously reported with young subjects [14]. Therefore, reliability of the simple rotary encoder method is verified in the elderly as well as in the young.

Relationships between the peak and mean values of the center of gravity transferring velocities during the STS movement measured by the rotary encoder and 3D motion analysis ranged from moderate to very high $(\mathrm{r}=0.60-0.77)$ for the cross and vertical directions but was low-moderate $(r=0.04$ and -0.47$)$ for the crosswise direction. Schenkman et al. [17] defined the STS movement as vertical transferring of the center of gravity. We previously reported that the vertical transferring phase of the center of gravity during the STS movement is closely related to leg strength, ADL ability, and risk for falls [18, 19]. From the results of the present study, we conclude that the center of gravity transferring velocity measured by the rotary encoder can evaluate the characteristics of the vertical transferring velocity of the center of gravity, and it may provide a simpler means for evaluating the STS movement ability in the elderly than 3D motion analysis. Moreover, moderate-to-high relationships were found not only with the vertical velocity but also with the cross velocity of the center of gravity. Kerr et al. [20] and Hughes et al. [21] reported that when transferring the center of gravity vertically during the STS movement, greatest ability and exertion are required in the phase from the starting movement to the hip lift, and some elderly people cannot achieve STS because of this large burden. In this phase, the elderly bend the trunk forward and the upper body shifts in the upper direction faster (Hughes et al., 1996; Kerr et al., 1997; Papa et al., 2000). Thus, they attempt to accomplish the STS movement using a reaction movement. The moderate relationship found between the velocities of the cross direction measured by the rotary encoder and 3D motion analysis may be related to this observation.

\section{Conclusion}

A rotary encoder appears to be useful for evaluating the center of gravity transferring velocity during the STS movement because it is highly correlated to the vertical velocity measured by $3 \mathrm{D}$ motion analysis.

\section{Acknowledgements}

The authors have no financial disclosures to make on this paper and contributed to all aspects of this paper and there is no sponsor to this study.

\section{References}

[1] P.O. Riley, M.L. Schenkman, R.W. Mann et al., Mechanics of a constrained chair-rise, Journal of Biomechanics 24 (1991) $77-85$.

[2] N.B. Alexander, A.B. Schultz, D.N. Warwick, Rising from chair: effect of age and functional ability on performance biomechanics, Journals of Gerontology Series A: Biological Sciences and Medical Sciences 46 (1991) M91-M98.

[3] D.W. vander Linden, D. Brunt, M.U. McCulloch, Variant invariant characteristics of the sit-to-stand task in healthy elderly adults, Archives of Physical Medicine and Rehabilitation 75 (1994) 653-660.

[4] C.A. Doorenbosch, J. Harlaar, M.E. Roebroeck et al., Two strategies of transferring from sit-to-stand; the activation of monoarticular and biarticular muscles, Journal of Biomechanics 11 (1994) 1299-1307.

[5] P.K. Schot, K.M. Knutzen, S.M. Poole et al., Sit-to-stand performance of older adults following strength training, Research Quarterly for Exercise and Sport 74 (2003) 1-8. 
[6] H. Hirschfeld, M. Thorsteinsdottir, E. Olsson, Coordinated ground forces exerted by buttocks and feet are adequately programmed for weight transfer during sit-to-stand, Journal of Neurophysiology 82 (1999) 3021-3029.

[7] Y.C. Pai, M.W. Rogers, Control of body mass transfer as a function of speed of ascent in sit-to-stand, Medicine and Science in Sport and Exercise 22 (1990) 378-384.

[8] D. Moxley Scarborough, D.E. Krebs, B.A. Harris, Quadriceps muscle strength and dynamic stability in elderly persons, Gait \& Posture 10 (1999) 10-20.

[9] M.M. Gross, P.J. Stevenson, S.L. Charette et al., Effect of muscle strength and movement speed on the biomechanics of rising from a chair in healthy elderly and young women, Gait \& Posture 8 (1998) 175-185.

[10] F. Mourey, T. Pozzo, I. Rouhier-Marcer et al., A kinematic comparison between elderly and young subjects standing up from and sitting down in a chair, Age. \& Ageing 27 (1998) 137-146.

[11] T. Mitsui, K. Zushi, Factors increasing stride length in the elderly during walking, with special reference to inverted pendulum movement, Japan Journal of Physical Education, Health and Sport Science 51 (2006) 447-457. (in Japanese)

[12] H. Okada, M. Ae, Body segment inertia parameter of the elderly and its application to motion analysis, Japanese Journal of Sport Science 15 (1996) 169-175. (in Japanese)

[13] T. Yokoi, R. Shibukawa, M. Ae, Body segment factor of Japanese children, Japan Journal of Physical Education, Health and Sport Science 31 (1986) 53-66. (in Japanese)

[14] T. Yamada, S. Demura, Reliability of center of gravity transfer velocity during the sit-to-stand movement and its relationship with leg muscle strength, Japan Journal of Test and Evaluation in Health and Physical Education 8 (2009) 33-38.

[15] U.P. Arborelius, P. Wretenberg, F. Lindberg, The effects of armrests and high seat heights on lower-limb joint load and muscular activity during sitting and rising, Ergonomics 35 (1992) 1377-1391.

[16] Y. Ebara, S. Yamamoto, Text of body dynamics: Analysis of sit to stand movement, Ishiyaku Publishers, Tokyo, 2001. (in Japanese)

[17] M. Schenkman, P.O. Riley, C. Pieper, Sit to stand from progressively lower seat heights -- alterations in angular velocity, Clinical Biomechanics 11 (1996) 153-158.

[18] T. Yamada, S. Demura, Relationships between ground reaction force parameters during a sit-to-stand movement and physical activity and falling risk of the elderly and a comparison of the movement characteristics between the young and the elderly, Archives of Gerontology and Geriatrics 48 (2009) 73-77.

[19] T. Yamada, S. Demura, The relationship of force output characteristics during a sit-to-stand movement with lower limb muscle mass and knee joint extension in the elderly, Archives of Gerontology and Geriatrics 50 (2010) e46-e50.

[20] K.M. Kerr, J.A. White, D.A. Barr et al., Analysis of the sit-stand-sit movement cycle in normal subjects, Clinical Biomechanics 12 (1997) 236-245.

[21] M. Hughes, D. Weiner, M. Schenkman et al., Chair rise strategies in the elderly, Clinical Biomechanics 9 (1994) 187-192.

[22] E. Papa, A. Cappozzo, Sit-to-stand motor strategies investigated in able-bodied young and elderly subjects, Journal of Biomechanics 33 (2000) 1113-1122. 